\title{
On the physical operation and optimization of the p-GaN gate in normally-off GaN HEMT devices
}

L. Efthymiou, ${ }^{1, a)}$ G. Longobardi, ${ }^{1}$ G. Camuso, ${ }^{1}$ T. Chien, ${ }^{2}$ M. Chen, ${ }^{2}$ and F. Udrea ${ }^{1}$

${ }^{1)}$ Department of Electrical Engineering, University of Cambridge, Cambridge, United Kingdom, CB3 OFA

${ }^{2)}$ Vishay General Semiconductor, New Taipei City, Taiwan, 23145

(Dated: 8 March 2017)

In this study an investigation is undertaken to determine the effect of gate design parameters on the on-state characteristics (threshold voltage, gate turn-on voltage) of pGaN/AlGaN/GaN HEMTs. Design parameters considered are pGaN doping and gate metal work function. The analysis considers the effects of variations in these parameters using a TCAD model matched with experimental results. A better understanding of the underlying physics governing the operation of these devices is achieved with a view to enable better optimization of such gate designs.

In the last decade an increased effort has been put on the development of power switches using $\mathrm{AlGaN} / \mathrm{GaN}$ HEMT structures. A two dimensional electron gas (2DEG) inherently exists at the $\mathrm{AlGaN} / \mathrm{GaN}$ heterointerface which creates a challenge when attempting the design of normally-off rather than normally-on devices. Nonetheless, as normally-off transistors are preferable in most power electronic applications several methods have been proposed which can lead to enhancement mode devices, among them the use of metal insulator semiconductor structures ${ }^{1}$, use of fluorine treatment ${ }^{2}$, recessed gate structures ${ }^{3}$ and use of a p-type cap layer ${ }^{456}$. Due to the relative maturity and controllability in the epitaxial growth of pGaN layers compared to the other techniques, $\mathrm{pGaN} / \mathrm{AlGaN} / \mathrm{GaN}$ HEMTs are considered the leading structure for commercialization. While several publications exist on different aspects of the performance of GaN devices (breakdown, buffer leakage, current collapse) ${ }^{78}$ there is less focus on the role that gate design parameters have in determining the on-state characteristics (threshold voltage, trans-conductance and gate turn-on current) of pGaN devices. These parameters are of great interest as problems such as unwanted device turn-on when the device is supposed to be off may occur in operation if threshold voltage is low. Secondly, gate turn-on may be a problem due to the non-insulated gate structure. Furthermore, several studies have reported that the gate stack and in particular the regions close to the Schottky contact/pGaN interface ${ }^{9}$ and the pGaN/AlGaN interface $^{10}$ can play an important role in the reliability of the device under forward bias stress. In the pGaN layer, the presence of defects related to Magnesium $(\mathrm{Mg})$ doping ${ }^{1011}$ and the generation of defects due to the presence of a high electric field ${ }^{9}$ can lead to an increase in the the leakage current via a defect percolation process and ultimately lead to device failure ${ }^{101213}$. An understanding of what affects parameters such as threshold voltage, trans-conductance, gate leakage and gate turn-on current is therefore paramount to achieving a good design. Some

\footnotetext{
a)Electronic mail: le257@cam.ac.uk
}

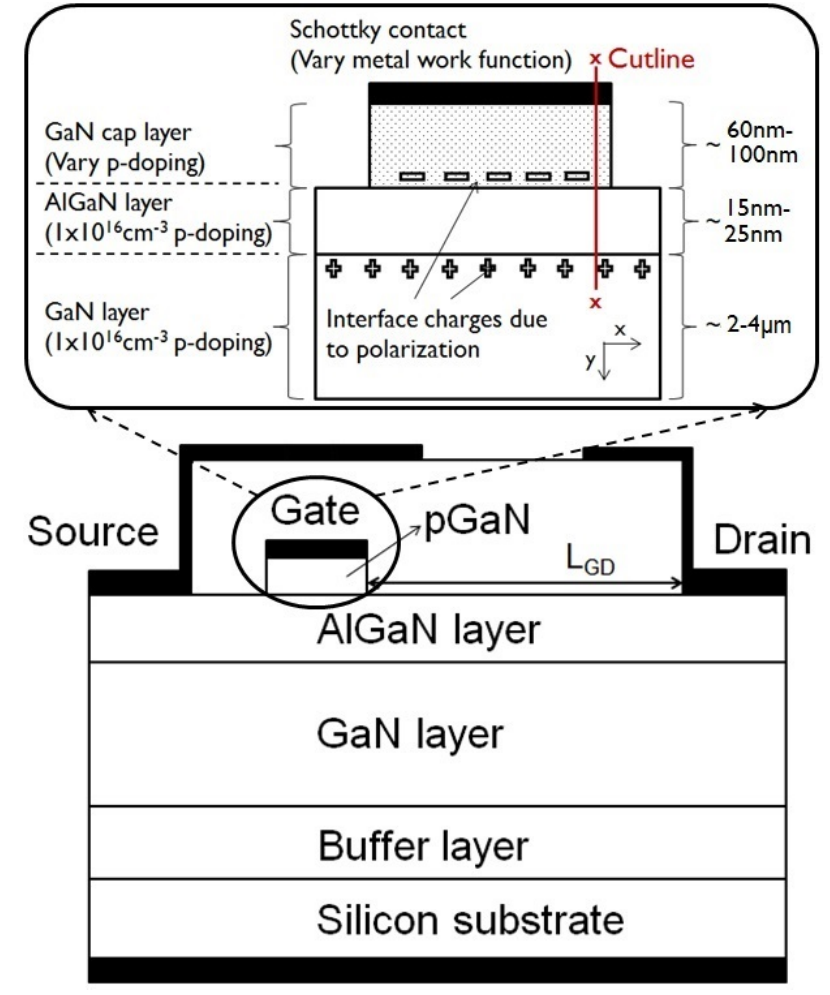

FIG. 1. Cross section of p-GaN/AlGaN/GaN HEMT device modelled.

literature exists on controlling these parameters, specifically on the impact that the gate metal used has on the on-state performance ${ }^{1415}$. While Hwang et al ${ }^{15}$ reports a large shift in threshold voltage when gate metal is varied, Lee et $\mathrm{al}^{14}$ does not. The disagreement between the two results can be explained when the different Schottky metal/pGaN barrier present in the two devices is taken into consideration and specifically relates to the scale of hole tunnelling current observed at this interface. The Schottky barrier observed is affected both by the gate metal work function and the acceptor doping used in the pGaN cap.

This study offers a comprehensive analysis of the un- 


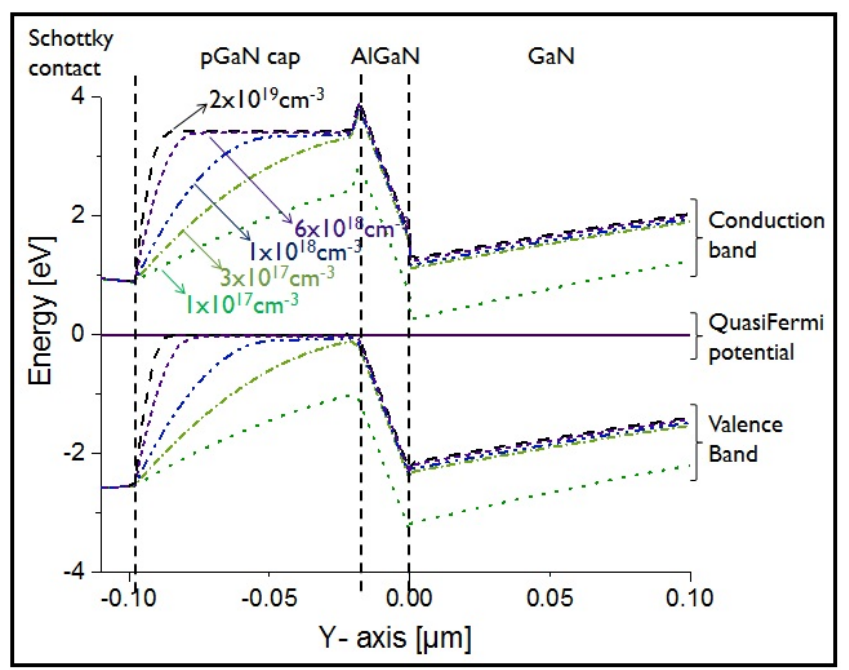

FIG. 2. Effect of pGaN gate doping variation on band diagram at zero bias.

derlying physics which governs the operation of the $\mathrm{pGaN} / \mathrm{AlGaN} / \mathrm{GaN}$ gate structure. A TCAD simulation model is matched thoroughly with device experimental measurements and is used to enable better understanding and optimization of such gate designs.

The device investigated is a lateral three-terminal device with an AlGaN/GaN heterostructure grown epitaxially on a standard silicon wafer (Fig. 1). A buffer layer is used to allow a high quality GaN layer to be grown despite the significant lattice mismatch between $\mathrm{GaN}$ and Si. Fixed charges were included in the TCAD simulation deck according to Ambacher et $\mathrm{al}^{16}$ to take into account the piezo-polarisation effect observed in $\mathrm{GaN}$ devices as was done in an earlier investigation ${ }^{17}$. A p-type doping of $1 \times 10^{16} \mathrm{~cm}^{-3}$ was added in the GaN layer to take into account the carbon doping as reported in literature ${ }^{18}$. Finally, a thin cap GaN layer was added to form the gate with a Magnesium $(\mathrm{Mg})$ p-type doping density of $2 \times 10^{19} \mathrm{~cm}^{-3}$. This doping value, also reported elsewhere in literature ${ }^{5}$, matched the experimental results. At room temperature $\mathrm{Mg}$ doping is not fully ionized under zero bias conditions given its energy level at $170 \mathrm{meV}-250 \mathrm{meV}$ above the valence band ${ }^{1519}$. Nonetheless, incomplete ionization does not affect the Schottky barrier and depletion region observed at the gate schottky metal/pGaN cap interface, both at zero bias and when the gate is biased, as activation of dopants occurs due to the presence of a large electric field. Therefore, in order to simplify the analysis the pGaN doping included in the TCAD model is fully ionized. However, this does not fully consider that part of the $\mathrm{Mg}$ atoms may be passivated by $\mathrm{Hy}-$ drogen $(\mathrm{H})$ atoms $^{20}$ or that self-compensation may occur due to deep donor states attributed to Nitrogen(N) vacancies $^{21}$. Nevertheless, it has been reported in literature that for $[\mathrm{Mg}]<3 \times 10^{19} \mathrm{~cm}^{-3}$ a doping efficiency in excess of $70 \%$ with a maximum net acceptor concentration of $1.8 \times 10^{19} \mathrm{~cm}^{-3}$ can be achieved ${ }^{11}$. Thus, considering the

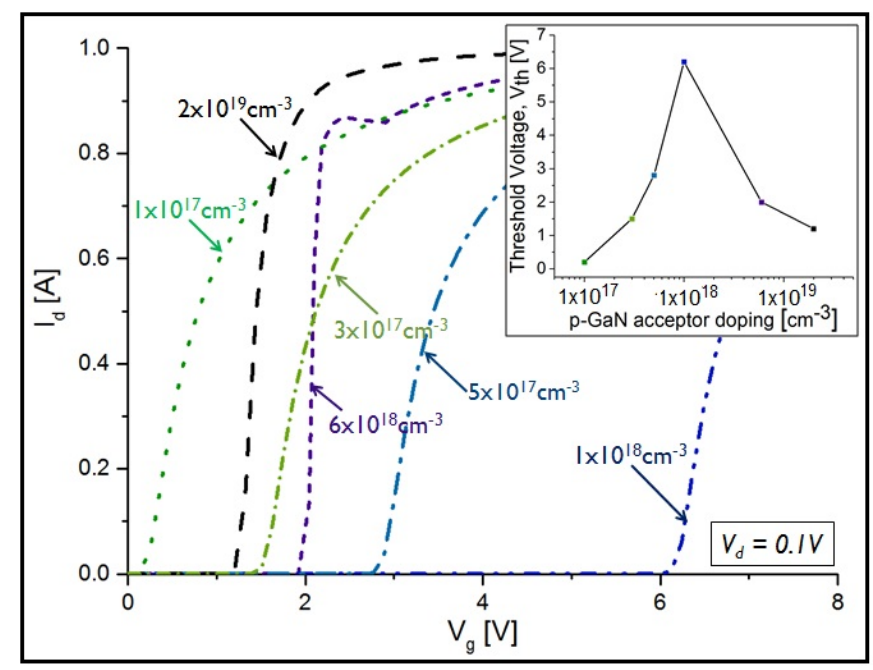

FIG. 3. $I_{D}-V_{G}$ transfer characteristic with variation in $\mathrm{pGaN}$ gate doping.

constant efforts to enhance effective doping in Mg-doped $\mathrm{GaN}^{192111}$, the simplification made here can be considered valid for the doping levels considered in this study. A more detailed look at the $\mathrm{p}-\mathrm{GaN} / \mathrm{AlGaN} / \mathrm{GaN}$ gate structure can be seen in Fig. 1. The p-GaN cap acceptor doping and metal work function were varied in the analysis.

The gate pGaN cap acceptor doping in the TCAD model was varied first. Looking at the conduction band along x-cutline (as shown in the inset of Fig. 1) at zero bias it can be observed that as the doping is increased a stronger depletion of the 2DEG beneath the gate is created as the conduction band moves further away from the Fermi level (see Fig. 2).

The variation in doping has a very significant effect on the threshold voltage of the device as seen in Fig. 3. As the doping initially increases, the threshold voltage of the device is also increasing (for doping values of $1 \times 10^{17} \mathrm{~cm}^{-3}$ to $1 \times 10^{18} \mathrm{~cm}^{-3}$ ) however as the doping is increased further the threshold voltage starts decreasing (for doping values $>6 \times 10^{18} \mathrm{~cm}^{-3}$ ).

To understand the variation of the threshold voltage with pGaN acceptor doping the band diagram when the gate is biased needs to be examined. When a positive gate bias voltage is applied this can lead to two effects:

- One effect is to modulate the 2DEG. When potential is applied at the p-GaN/AlGaN/GaN junction, as seen in region (i) of Fig. 4, the energy of electrons at the $\mathrm{AlGaN} / \mathrm{GaN}$ interface increases so the conduction band moves closer to the eFermi level forming the 2DEG.

- The other effect occurs at the Schottky/p-GaN interface which can be seen in region (ii) of Fig. 4. As the gate bias voltage is increased the junction is reverse biased and the depletion from the Schottky contact extends into the pGaN. 


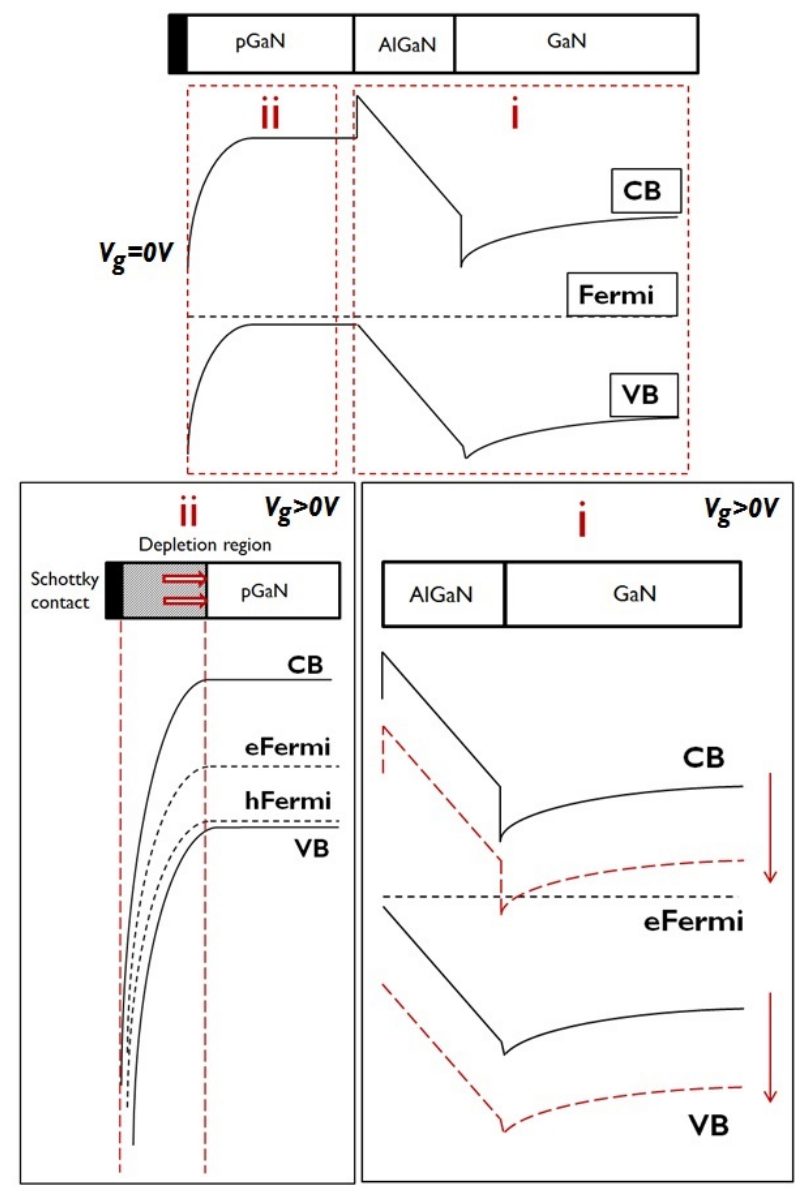

FIG. 4. Effect of gate bias on different sections of the gate structure band diagram.

The potential drop across this depletion region, when the gate is positively biased, is higher at increased values of the pGaN cap acceptor doping. This translates into the need for a higher gate bias voltage in order to modulate the 2DEG and is what causes the threshold voltage of the device to initially increase when pGaN doping is increased.

However, at a sufficiently high acceptor doping in the pGaN cap the potential barrier for holes at the Schottky contact/pGaN interface becomes very narrow and this can lead to tunnelling of holes through the barrier. At this point, no further depletion of the pGaN layer occurs when the gate voltage bias is increased. Gate potential applied is no longer dropped across the depletion region in the pGaN but contributes to the shifting of the AlGaN/GaN interface conduction band towards the Fermi level and thus the formation of the 2DEG at a lower gate bias voltage. Note that the pGaN layer is electrically connected to the gate in this regime. Therefore, the threshold voltage is lowered $\left(V_{t h}<2 V\right)$ at high pGaN doping values $\left(>6 \times 10^{18} \mathrm{~cm}^{-3}\right)$ instead of increasing further as was the trend observed initially. This analysis is illustrated best when looking at Fig. 5 which compares the band diagrams for a doping of $1 \times 10^{18} \mathrm{~cm}^{-3}$ (negligi-

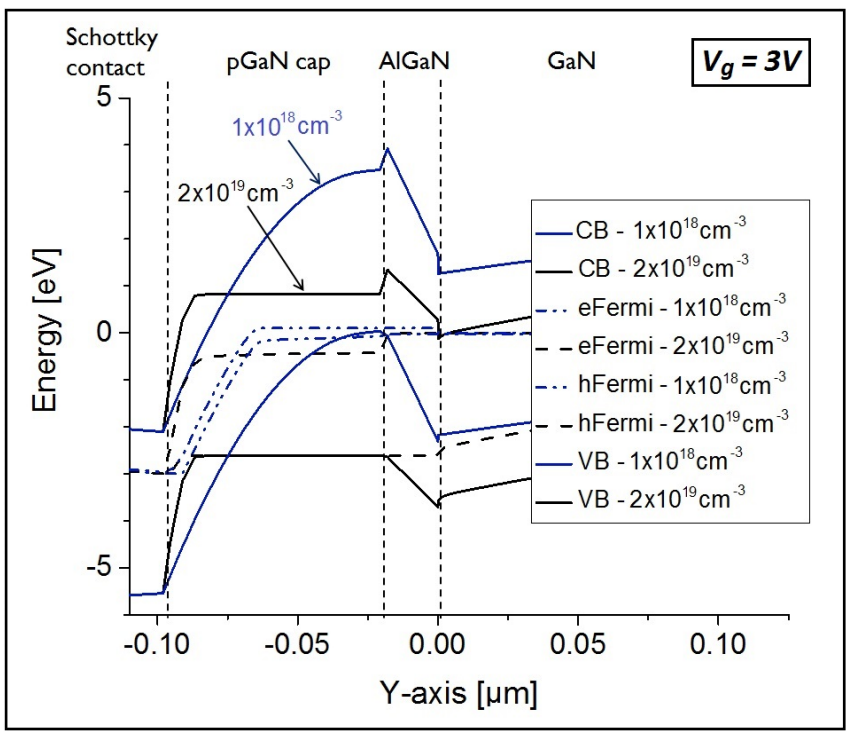

FIG. 5. Gate structure band diagram at gate bias of $3 \mathrm{~V}$ for pGaN doping of $1 \times 10^{18} \mathrm{~cm}^{-3}$ (negligible hole tunnelling) and $2 \times 10^{19} \mathrm{~cm}^{-3}$ (considerable hole tunnelling).

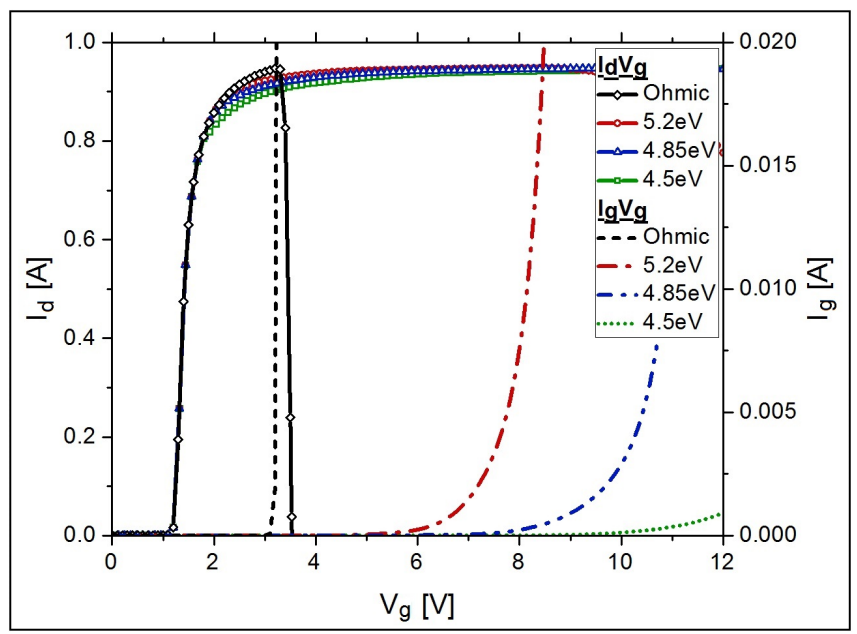

FIG. 6. $I_{D}-V_{G}$ transfer characteristic and gate turn-on as gate metal work function is varied.

ble hole tunnelling current) and $2 \times 10^{19} \mathrm{~cm}^{-3}$ (considerable hole tunnelling current) at a gate bias voltage of $3 \mathrm{~V}$. This analysis is further verified by not including the hole tunnelling model in the TCAD simulations. In this scenario the threshold voltage keeps increasing as the doping is increased instead of decreasing when a pGaN doping value above a certain level is used. The critical pGaN acceptor doping level needed for substantial tunnelling to occur is dependent on the gate metal work function and the effective hole mass. The latter has been fixed to $0.3 m_{e}$ according to Santic et $\mathrm{al}^{2223}$. A reduced gate metal work function would lead to a higher and narrower Schottky potential barrier for holes where increased tunnelling can be observed. 




FIG. 7. Gate structure band diagram at gate bias of $\mathrm{Vg}=3 \mathrm{~V}$, $9 \mathrm{~V}>\mathrm{Vth}$ for TCAD model with pGaN doping of $2 \times 10^{19} \mathrm{~cm}^{-3}$.

Very importantly what this analysis also reveals is that above a certain pGaN doping level, variations in Schottky contact work function have a limited effect on the threshold voltage of the device (see Fig. 6 - TCAD model pGaN doping: $2 \times 10^{19} \mathrm{~cm}^{-3}$ ) which can explain the observations in the study by Lee et $\mathrm{al}^{14}$ where a high pGaN doping of $5 \times 10^{19} \mathrm{~cm}^{-3}$ is used.

A model with an ohmic gate contact was also simulated and shows an identical threshold voltage to that given by the Schottky metal/high p-GaN doping models (see Fig. 6 ). This again reinforces the argument that a high level of tunnelling occurs at the Schottky/pGaN interface.

The effect of pGaN doping and gate metal work function on the gate turn-on voltage is also of interest in order to achieve as wide a gate bias operating range as possible. The band diagram at the $\mathrm{AlGaN} / \mathrm{GaN}$ interface was analyzed as the gate terminal was biased to higher voltages (i.e. at $\mathrm{Vg}>3 \mathrm{~V}>\mathrm{Vth}$ ). The analysis was undertaken for the TCAD model with high pGaN acceptor doping $\left(2 \times 10^{19} \mathrm{~cm}^{-3}\right)$ as this matched the experimental device data.

Below the threshold voltage, the conduction band shifts towards the Fermi level with no electric field change across the AlGaN layer (see Fig. 4(i)). This occurs up to the point where the Fermi level is pinned at the AlGaN/GaN interface (see Fig. 7). As gate bias voltage is increased further, the barrier at the $\mathrm{AlGaN} / \mathrm{pGaN}$ interface starts to be lowered both for electrons flowing from the 2DEG to the gate contact and holes flowing away from the pGaN. This is illustrated by observing the band diagram at two different gate bias conditions $(\mathrm{Vg}=3 \mathrm{~V}$, $9 \mathrm{~V})$ as seen in Fig. 7. The majority of gate turn-on current is comprised of electrons flowing from the source contact to the gate contact. The following interesting observations can be made:

- The use of a Schottky contact compared to an ohmic contact leads to a gate turn-on at a higher gate bias (see Fig. 6). With a Schottky gate contact a higher bias voltage is needed to reduce the potential barrier at the $\mathrm{pGaN} / \mathrm{AlGaN}$ interface as a potential drop is also observed across the pGaN cap layer depletion region. The use of a Schottky barrier in order to achieve a wide operating gate bias range is therefore essential and contradicts the suggestion in the study by Chang et $\mathrm{al}^{24}$ regarding the use of an ohmic contact.

- Gate turn-on in this device is at a gate bias $>8 \mathrm{~V}$ which allows a reasonably wide range of operation.

- A higher gate metal work function will lead to a lower gate turn-on voltage (see Fig. 6). This agrees with the observation made by Hwang et al ${ }^{15}$.

- For high pGaN doping values $\left(>1 \times 10^{19} \mathrm{~cm}^{-3}\right)$, an increase in the acceptor doping will not affect the threshold voltage of the device but will affect the gate turn-on voltage.

In conclusion, TCAD simulations have been used to enable a thorough understanding of the operation of the gate structure of the pGaN cap E-HEMT. Some very important design considerations are summarized below.

- As pGaN doping initially increases the threshold voltage of the device is also increasing (for doping values of $1 \times 10^{17} \mathrm{~cm}^{-3}$ to $1 \times 10^{18} \mathrm{~cm}^{-3}$ ) however, as the doping is increased further the threshold voltage starts decreasing (for doping values $\left.>6 \times 10^{18} \mathrm{~cm}^{-3}\right)$. This is due to hole tunnelling at the metal/pGaN interface establishing a tight electrical connection between the gate metal and the pGaN layer.

- At high doping levels, the threshold voltage cannot be significantly altered by the use of a different gate metal. This finding provides clarity to the observations in the study by Lee et $\mathrm{al}^{14}$.

- A variation in gate metal used affects the gate turnon voltage of the device. This agrees with the observation in the study by Hwang et $\mathrm{al}^{15}$.

- The use of a Schottky gate contact rather than an ohmic gate contact is essential in order to achieve a wide operating gate bias range. This finding contradicts the suggestion given in the study by Chang et $\mathrm{al}^{24}$.

This study reveals the trends that need to be taken into consideration when designing the gate characteristics such that the gate operation range is maximized and the device operates in an optimal way. 
${ }^{1}$ T. Oka and T. Nozawa, IEEE Electron Device Lett., 29, 668 (2008).

${ }^{2}$ Y. Cai, Y. Zhou, K. J. Chen, and K. M. Lau, IEEE Electron Device Lett., 26, 435 (2005)

${ }^{3}$ W. Saito, Y. Takada, M. Kuraguchi, K. Tsuda, and I. Omura, IEEE Trans. Electron Devices, 53, 356, (2006).

${ }^{4}$ Y. Uemoto, M. Hikita, H. Ueno, H. Matsuo, H. Ishida, M. Yanagihara, T. Ueda, T. Tanaka, and D. Ueda, IEEE Trans. Electron Devices, 54, 3393 (2007).

${ }^{5}$ I. Hwang, H. Choi, J. Lee, H. S. Choi, J. Kim, J. Ha, C. Y. Um, S. K. Hwang, J. Oh, J. Y. Kim, J. K. Shin, Y. Park, U. I. Chung, I. K. Yoo, and K. Kim, Proc. ISPSD, Bruges, Belgium, p.41 (2012).

${ }^{6}$ L. Y. Su, F. Lee, and J. J. Huang, IEEE Trans. Electron Devices, 61, 460 (2014).

${ }^{7}$ S. Kaneko, M. Kuroda, M. Yanagihara, A. Ikoshi, H. Okita, T. Morita, K. Tanaka, M. Hikita, Y. Uemoto, S. Takahashi, and T. Ueda, Proc. ISPSD, Hong Kong, p.41 (2015).

${ }^{8}$ M. H. Kwan, K. Wong, Y. S. Lin, F. W. Yao, M. W. Tsai, Y. Chang, P. C. Chen, R. Y. Su, C. Wu, J. L. Yu, F. J. Yang, G. P. Lansbergen, H. Wu, M. Lin, C. B. Wu, Y. Lai, C. Hsiung, P. Liu, H. Chiu, C. Chen, C. Y. Yu, H. S. Lin, M. Chang, S. Wang, L. C. Chen, J. L. Tsai, H. C. Tuan, and A. Kalnitsky, Proc. ISPSD, Hawaii, p. 17 (2014).

${ }^{9}$ M. Meneghini, I. Rossetto, V. Rizzato, S. Stoffels, M. Van Hove, N. Posthuma, T. Wu, D. Marcon, S. Decoutere, G. Meneghesso, and E. Zanoni, 5, 14 (2016).

${ }^{10} \mathrm{M}$. Tapajna, O. Hilt, E. Bahat-Treidel, J. Wurf, and J. Kuzmik, IEEE Electron Device Lett., 37, 385 (2016).

${ }^{11}$ A. Castiglia, J. F. Carlin, and N. Grandjean, Appl. Phys. Lett., 98, 213505-1 (2011).
${ }^{12}$ M. Tapajna, O. Hilt, E. Bahat-Treidel, J. Wurfl, and J. Kuzmik, Appl. Phys. Lett., 107, 193506-1 (2015)

${ }^{13}$ A. N. Tallarico, S. Stoffels, P. Magnone, N. Posthuma, E. Sangiorgi, S. Decoutere, and C. Fiegna, IEEE Electron Device Lett., 3106, 38, 99 (2016).

${ }^{14}$ F. Lee, L. Y. Su, C. H. Wang, Y. R. Wu, and J. Huang, IEEE Electron Device Lett., 36, 232 (2015).

${ }^{15}$ I. Hwang, J. Kim, H. S. Choi, H. Choi, J. Lee, K. Y. Kim, J. B. Park, J. C. Lee, J. Ha, J. Oh, J. Shin, and U. I. Chung, IEEE Electron Device Lett., 34, 202 (2013).

${ }^{16}$ O. Ambacher, J. Smart, J. R. Shealy, N. G. Weimann, K. Chu, M. Murphy, W. J. Schaff, L. F. Eastman, R. Dimitrov, L. Wittmer, M. Stutzmann, W. Rieger, and J. Hilsenbeck, J. Appl. Phys., 85, 3222 (1999).

${ }^{17}$ L. Efthymiou, G. Longobardi, G. Camuso, F. Udrea, E. Lin, T. Chien, M. Chen, Proc. ISPSD, Prague, Czech Republic, p.71 (2016).

${ }^{18}$ M. J. Uren, J. Moreke, and M. Kuball, IEEE Trans. Electron Devices, 59, 3327 (2012).

${ }^{19}$ M. Oh, J. J. Lee, J. K. Lee, and H. Kim, J. Alloys Compd., 585 , 417 (2014).

${ }^{20}$ S. Nakamura, N. Iwasa, M. Senoh and T. Mukai, Jpn. J. Appl. Phys., 31, 1258 (1992).

${ }^{21}$ J.-D. Hwang, Z.-Y. Lai, C.-Y. Wu, and S.-J. Chang, Jpn. J. Appl. Phys., 44, 1726 (2005).

${ }^{22}$ B. Santic, Semicond. Sci. Technol., 18, 219 (2003).

${ }^{23}$ A. Salvador, G. Liu, W. Kim, . Aktas, A. Botchkarev, and H. Morko, Appl. Phys. Lett., 67, 3322 (1995).

${ }^{24}$ T. Chang, T. Hsiao, C. Huang, W. Kuo, S. Lin, G. S. Samudra and Y. C. Liang, IEEE Transactions on Electron Devices, 62, 339 (2015). 